\title{
Effect of Nanograin Sublayers on Structure and Properties of the Electrodeposited ZnO Arrays
}

\author{
V. V. IVANOV1', G. V. TSEPILOV ${ }^{1}$, I. V. IYENYUTIN², and A. N.VOROPAY1 \\ 'Technocomplekt closed joint stock company, Shkol'naya str., 10a, Dubna, \\ Moscow area (oblast), 141981 Russia. \\ ${ }^{2}$ Turboenergoremont, Leninskiy prospect 114, block. A, premises $170 \mathrm{H}$, \\ Saint Petersburg, Leningradskaya area (oblast), 198207, Russia. \\ *Corresponding author E-mail: ivanov @ techno-com.ru \\ http://dx.doi.org/10.13005/ojc/320233
}

(Received: February 15, 2016; Accepted: March 28, 2016)

\begin{abstract}
The influence of $\mathrm{ZnO}$ nanograin sublayers deposited at the initial stage of the electrodeposition on morphology, structure, optical and electrical properties of the nanostructured arrays of this material produced in the pulsed electrodeposition mode has been investigated. It is shown that the formation of these sublayers contributes to enlargement of the crystal grains and the formation of separate zinc oxide nanorods, which are perpendicular to the surface of the substrate. Moreover, the zinc oxide array electrodeposited in the pulsed mode with using nanograin sublayers has higher specific resistance, better transparency, scatters less visible light and has an appropriate optical band gap.
\end{abstract}

Keywords: pulsed electrodeposition, 1-D ZnO, structure, optical properties, AFM.

\section{INTRODUCTION}

Zinc oxide $(\mathrm{ZnO})$ belongs to the rapidly developing class of materials used for electronic and optoelectronic applications ${ }^{1-9}$. The reason is not only in the unique properties of this wide bandgap semiconductor $(\mathrm{Eg}=3.37 \mathrm{eV})$ with direct optical transitions and a large exciton binding energy $(60 \mathrm{MeV}$ at the room temperature), but also in the possibility of obtaining $\mathrm{ZnO}$ using different technologies, including inexpensive and suitable for large-scale production of liquid-phase chemical and electrochemical methods $^{2-10}$. The another advantage of the material $\mathrm{ZnO}$ is its propensity to form nanostructures with different morphology among which the greatest interest for the developers of electronic products and optoelectronics attract nanowires and nanorods, i.e., one-dimensional (1-D) nanostructures of zinc oxide. The method of electrochemical deposition from aqueous solutions allows producing arrays of 1-D 
$\mathrm{ZnO}$ within 10 - 90 minutes on the large areas and at a temperature not exceeding $100^{\circ} \mathrm{C}^{2-10}$. The efficiency of this method depends also by using readily available dilute solutions of zinc salts and sodium (or potassium) as starting materials. They typically do not contain modifying additives of complexing agents or surface-active organic substances that pollute the environment. The success of the method of electrodeposition is in the fabrication of optoelectronic devices, gas nanosensors, UV detectors, solar cells organic and hybrid photovoltaic cells is due to the fact that its use for the creation of nanostructures of zinc oxide combines such advantages as the simplicity of the technological equipment and the suitability for mass production with a good control of the process. In order to make a necessary morphology for the $\mathrm{ZnO}$ nanostructures, scientists $^{2,10}$ widely investigate the influence of cathode potential, the electrolyte composition, the temperature and the time of deposition, the presence or absence in the solution of various organic additives and complexes, the following hydrothermal treatments, the annealing and even the gravity on the structure and properties of electrodeposited arrays of zinc oxide. The electrolysis process is carried out in potentiostatic or galvanostatic conditions, i.e. at the constant potentials of the cathode or cathode current, respectively2-4,8. However, as was shown in ${ }^{5-7,9-10}$, the pulsed electrolysis provides a controlled growth of semiconductor nanostructures without the threat of contamination of organic or other impurities from the electrolyte. In ${ }^{5-7,9-10}$ was demonstrated the possibility of controlling the morphology of the electrodeposited in pulsed arrays of zinc oxide in the same galvanic bath by varying the modes of cathodic pulsed electrochemical deposition of $\mathrm{ZnO}$ without rotation of the substrates and changes in the composition of the electrolyte.

Since the formation of 1-D $\mathrm{ZnO}$ in the electrodeposition is carried out by means of selforganization (the bottom-up analysis), the most important is the initial phase of the process, when on the surface of the foreign substrate the first crystal nuclei are formed. Therefore a sufficient attention was given for the creation of nanostring sublayers that are orienting the further growth of nanorods and nanowires of $\mathrm{ZnO}$, although all the works ${ }^{2-4,8}$ dealt with the electrolysis at constant currents or potentials. In this work for the first time examines the impact of nanograin sublayers of $\mathrm{ZnO}$ that are created at the initial stage of electrodeposition for the morphology, structure, optical and electrical properties of nanostructured arrays of zinc oxide, which are produced in the pulsed electrodeposition.

\section{The methodology of the experiment}

The fabrication of arrays of nanorods of zinc oxide was performed by the pulsed cathodic electrochemical deposition in a thermostated threeelectrode electrochemical cell with the non-mixed aqueous electrolyte containing of $0.01 \mathrm{M} \mathrm{Zn}\left(\mathrm{NO}_{3}\right)_{2}$ and $0.1 \mathrm{M} \mathrm{NaNO}_{3}$ at a temperature of $70^{\circ} \mathrm{C}$. As substrates (cathode, or working electrode) were used plate brand FTO TEC 7 Pilkington company, USA. A platinum spiral served as a counter-electrode and as the reference electrode a saturated silver chloride electrode $\mathrm{Ag} / \mathrm{AgCl}$. In the manufacture of the ZnOsp sample (sp - seed and pulse), at the first stage of electrodeposition was created a nanograin $\mathrm{ZnO}$ sublayer by feeding in the same electrolyte at the FTO working electrode a constant potential of $U=-1.3 \mathrm{~V}$ during 30 seconds. Then, using the impulse potentiostate PI-50-1.1, equipped with a programmer PR-8, for the implementation of pulse electrolysis for 10 minutes at a substrate-cathode were applied square waves of potential: the lower limit $U_{\text {off }}$ cathode potential was -0.7. In, the upper limit Uon $=-1.3 \mathrm{~V}$ (the potentials are given relative to the reference electrode $\mathrm{Ag} / \mathrm{AgCl}$ ). The duty cycle, i.e. the ratio of pulse duration to the sum of impulse duration and pauses, and the frequency as the reciprocal of the sum of the durations of the pulse and the pause, were 0.4 and $2 \mathrm{~Hz}$, respectively. A sample of zinc oxide by $\mathrm{ZnOp}$ ( $\mathrm{p}$ - a pulse) was electrodepositioned in a same pulsed mode, and ZnOsp, but at the initial stage of its manufacture we did not create the nuclei sublayers using of potentiostatic electrolysis.

The study of the optical properties of nanostructured layers of zinc oxide was performed by a spectrophotometer SF-2000, which is equipped with a console of a mirror and diffuse reflection SFD2000. As control samples during the registration of the spectrum of optical transmission $T(\lambda)$ was used the FTO substrate. The optical width of the forbidden zone $E_{g}$ of $\mathrm{ZnO}$ layers was determined similarly to that described in ${ }^{10}$ by the extrapolation to the energy axis of the linear section of the dependence of $[-\ln (T) \cdot h v]^{2}$ against $h v$. The disordered structure 
of $\mathrm{ZnO}$ was evaluated by the value of Urbach energy $E_{o}$, which is determined by the angle of inclination of the linear section of the dependence of $\ln [-\ln (T)]$ against $h v$. The light scattering factor $(\mathrm{Hf}$, Haze factor) was calculated according to ${ }^{10}$ as the ratio of diffuse reflectance to the total reflection $\mathrm{R}$ (the sum of diffuse and specular reflections).

To analyze the structural and substructural parameters of arrays of $\mathrm{ZnO}$ the X-ray spectra (XRD) were recorded using diffractometer DRON-4 in the radiation $\operatorname{CoK}_{\alpha}\left(\lambda_{\text {CoK } \alpha}=1.7889 \AA\right)$. Scanning was carried out with focusing according to Bragg-Brentano $(\theta-2 \theta)$. Processing of the obtained X-ray diffraction patterns (background separation, the separation of the doublet $K_{\alpha 1}-K_{\alpha 2}$ etc.), as well as the calculation of profile of diffraction lines were performed using programs "New_Profile v.3.4 (486)" and "OriginPro v.7.5". The presence of crystalline phases was detected by comparing data of the experimental $\mathrm{X}$-ray diffraction patterns with the JCPDS database of reference data using the program "PCPDFWIN v.1.30". Evaluation of the regions of coherent scattering (RCS) and microstresses $\Delta d / d$ (where $d$ is the period of the crystalline lattice according to JCPDS, $\Delta d$ is the difference between experimental and reference values of the period lattice) in arrays of zinc oxide was carried out by analyzing the broadening of $\mathrm{X}$-ray diffraction peaks, with taking into the consideration the presence of the instrumental broadening by the method of approximations of Williamson Hall according to ${ }^{11,12}$. The residual stress in $\mathrm{ZnO}$ was determined according to the methodology that was proposed in ${ }^{11}$. To study the texture of electrodeposited arrays of zinc oxide by the method of Harris we used the values of integral intensities of X-ray diffraction peaks, in accordance with ${ }^{13}$. For each peak, we calculated the value of the pole density $P(h k l)$, which characterizes the probability with which the normal to the surface of the crystallite coincides with the normal to the plane ( $h k l)$, in another word, it determines the number of crystallites, and their plane ( $h k l)$ is parallel to the sample surface. A pole density was calculated for all reported X-ray diffraction peaks, the values of $\mathrm{P}(h k l)>1$ was attributed to the axes of the texture.

The study of surface morphology of arrays of zinc oxide produced by the method of semicontact atomic force microscopy (AFM) on the device "Nanolaboratory NTEGRA prima, NT-MDT".

1. glass; 2-FTO; 3 - nuclei ZnO sublayer; 4. ZnO nanorods; 5 - contact In coated with "Contactol"; 6. copper wire contact; 7 - copper wire.

To determine the type of conductivity of $\mathrm{ZnO}$ has been used the standard method of using a thermal probe14. To study the electrical properties of electrodeposited arrays of zinc oxide as vertically arranged on the FTO substrates $\mathrm{ZnO}$ nanorods, were fabricated metal contacts with Indium (In). Indium was chosen as the contact material because of its ratio of work output $\ln \left(\theta_{\ln }=4.09 \mathrm{eV}\right)$ and the electron affinity to $\mathrm{ZnO}\left(x_{\mathrm{ZnO}}=4.2 \mathrm{eV}\right)$, and it must ensure the formation of ohmic contacts with $\mathrm{ZnO}$, according

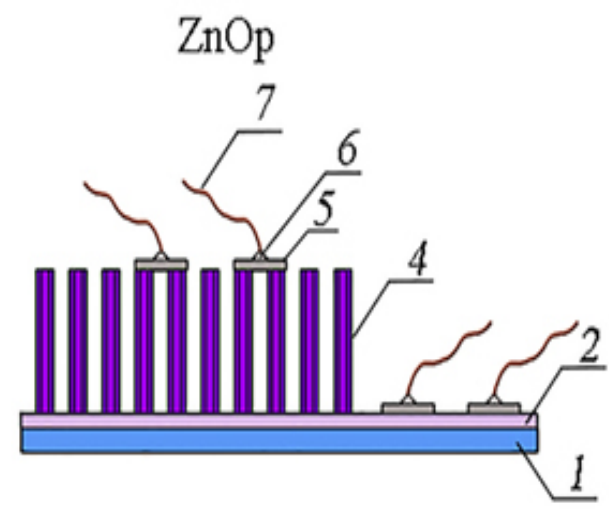

ZnOsp

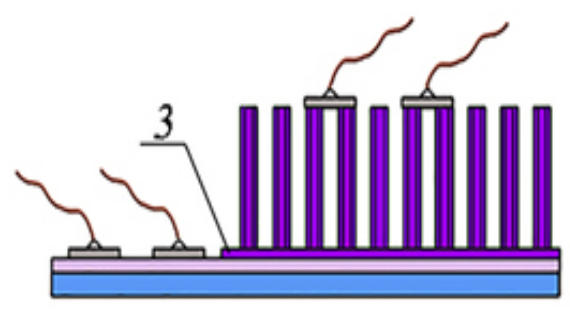

Fig. 1: A schematic representation of test samples with nanostructured arrays of $\mathrm{ZnOsp}$ (without a nuclei sublayer) and $\mathrm{ZnOsp}$ (with a nuclei $\mathrm{ZnO}$ sublayer) 
to ${ }^{1-15}$, In order to avoid shunting, In vacuum deposition was performed under an angle of $70^{\circ}$ to the surfaces of $\mathrm{ZnO}$ and FTO in their limited areas $(0.1 \times 1.0 \mathrm{~cm})$ using a removable shadow mask made of aluminum. Then, on the surface of Indium was applied a conductive adhesive with a silver filler "Contactol" to attach to In copper microwires. The test samples which were manufactured in this manner and presented in figure 1 were used for measuring dark current-voltage characteristics (CVC) on the diagram of a curve tracer ${ }^{16}$ by connecting to a commercial curve tracer L2-56 for a direct visualization of the CVC on the screen.

According to the results of CVC, measured in the case of the location of two Indium contacts on the surface of FTO, was obtained the total resistance $R_{t}=U / l$ and was calculated resistivity $P_{\mathrm{FTO}}$ of FTO transparent conductive layer according to the formula:

$$
\rho F T O=R t \cdot S F T O / w
$$

where $S_{F T O}-$ multiplication of the length of the Indium contact to the FTO layer thickness; $w$ is the distance between two Indium contacts disposed on FTO. By using the CVC we obtained a value of pFTO $=3.5 .10-4 \Omega . c m$, which corresponds to the data of the certificate for FTO of a brand of TEC 7 Pilkington company, USA. Calculations of the resistivity of the nanorods of zinc oxide $p_{Z n O}$ were based on measurements of CVC for Indium contacts between the top ends of the nanorods of zinc oxide, and FTO:

$$
U / I=R \Sigma=\rho F T O \cdot L / S F T O+2 \cdot \rho Z n O \cdot t / S c
$$

where $R_{\Sigma}$ is the total resistance derived according to CVC between two Indium contacts, one of them was located on FTO and the other was deposited on the upper ends of the nanorods of zinc oxide; $\mathrm{L}$ - the distance between these contacts; $\mathrm{t}$ - the average length of the $\mathrm{ZnO}$ nanorod $(\mathrm{t} \approx 0.4 \mu \mathrm{m}) ; S_{c}-$ the Indium contact area; the coefficient 2 takes into account the total area of cavities between the $\mathrm{ZnO}$ nanorods. If both contacts were located on the upper ends of the nanorods of zinc oxide, the obtained from the corresponding CVC the ratio $\mathrm{U} / \mathrm{I}$ correspond to the expression:

$$
U / I=R \Sigma=\rho F T O \cdot l / S F T O+4 \rho Z n O \cdot t / S c
$$

where $I$ - is the gap between the centers of the Indium contacts. The study of the temperature dependence of the electrical conductivity of electrodeposited in a pulsed mode of $\mathrm{ZnO}$ nanostructured layers for measurement of their activation energy $E_{a}$ was performed by analogy with the described in ${ }^{17}$ by using a measuring stand, providing a temperature control of test samples in air atmosphere and measuring the change in the resistance of $\mathrm{ZnO}$ by decreasing the thermostat temperature and of the investigated sample in the range from 150 to $20^{\circ} \mathrm{C}$. Depending on $\mathrm{lg} /$ from $1 / T$ (wherel is the current $(A)$ flowing through the $\mathrm{ZnO}$ nanorods at a fixed voltage of $1.0 \mathrm{~V}$; $\mathrm{T}$ is the temperature $(\mathrm{K})$ ) we determined the activation energy using the formula:

$$
\operatorname{tg} \alpha=-0.43 \cdot E a / k
$$

where $E_{\alpha}-$ an activation energy, $\mathrm{j} ; k-$ Boltzmann constant, j/K.

\section{RESULTS AND DISCUSSION}

Figure 2 shows the $\mathrm{X}$-ray diffraction pattern produced in the pulse regimen of the electrodeposition of nanostructured arrays of zinc oxide, obtained without the use of nuclei sublayer ( $\mathrm{ZnOp})$ and with using of nuclei sublayer ( $\mathrm{ZnOsp}$ ).

Their analysis indicates that both arrays of $\mathrm{ZnO}$ are single-phase and have a hexagonal structure of the wurtzite modification ( $\mathrm{ZnO}$, JCPDS PDF\#361451). The electrodeposited arrays of zinc oxide are nanocrystalline, and the area of coherent dispersion of $\mathrm{ZnO}$ ठ sample are in the range of 60 to $115 \mathrm{~nm}$, and in the case of use in the electrodeposition of nuclei sublayers of area of coherent dispersion increase and range from 80 to $200 \mathrm{~nm}$, which indicates to an improvement of the structure. Both samples characterized by low microstresses $\Delta / d \approx 10^{-3}$, the compressive residual stress of 0.1 GPA is for ZnOp and 0.4 GPA for ZnOsp. 
According to the calculations, unlike $\mathrm{ZnOp}, \mathrm{ZnOsp}$ sample is characterized by larger crystal lattice parameters along the $c$ axis $(c=5.22$ $\AA$ ) compared to the reference $\mathrm{ZnO}$ (according to JCPDS PDF\#361451, C = 5.207 ̊). On the other hand, the array of $\mathrm{ZnOp}$ is weakly textured in the direction $<001>$, i.e. in a perpendicular direction to the substrate surface $(P(002)=1.6)$, while the sample ZnOsp, fabricated with electrodeposition of the nuclei sublayer at the initial stage of growth, has

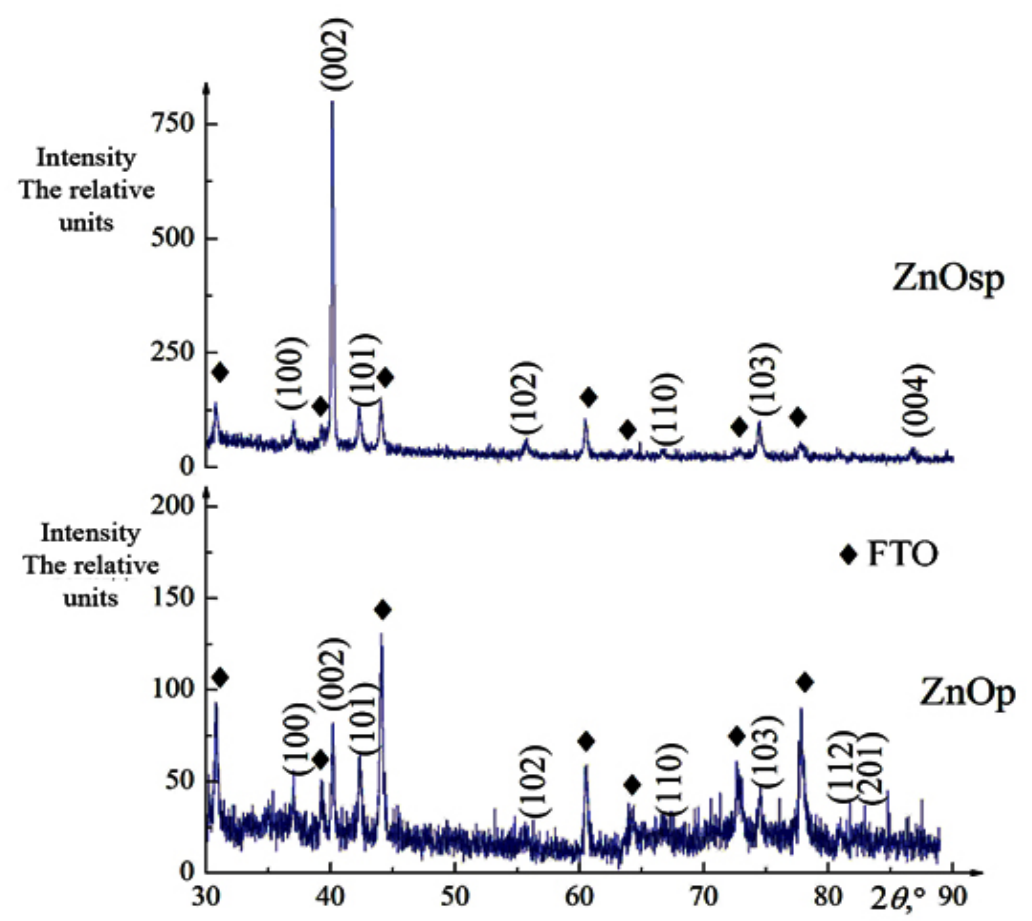

Fig. 2: The X-ray diffraction pattern of nanostructured arrays of zinc oxide manufactured without the use of nuclei sublayer (ZnOp) and with the using of nuclei sublayer (ZnOsp)
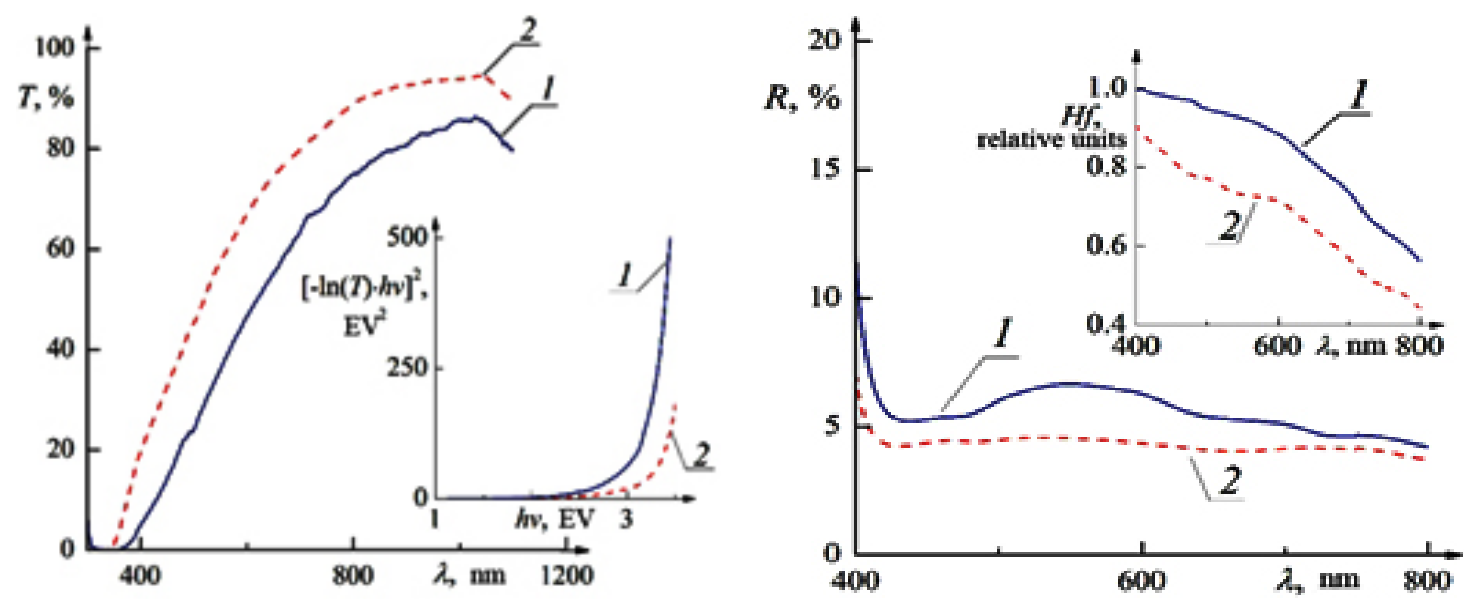

Fig. 3: Optical properties of nanostructured arrays of zinc oxide manufactured without the use of nuclei sublayer (ZnOp) and with the use of nuclei sublayer (ZnOsp): optical transmittance spectrum $\mathrm{T}(\lambda)(\mathrm{a})$, an insert - a schedule to determine Eg; optical reflection spectrum $\mathbf{R}(\lambda)(\mathrm{b})$, an insert - a range of the light scattering factor of $\mathrm{Hf}(\lambda) 1-\mathrm{ZnOp} ; 2-\mathrm{ZnOsp}$ 
a significant preferential orientation perpendicular to the substrate, as it has an axial texture of $(002)=3.2$. Optical properties of electrodeposited nanostructured arrays of $\mathrm{ZnO}$ in a pulsed mode (figure 3 ) show a sufficiently high transparency and an evident absence of an interference pattern of 1-D $\mathrm{ZnO}$ in the optical transmission spectrum $\mathrm{T}(\lambda)$ (figure 3A).

The width of the forbidden zone for direct optical transitions in $\mathrm{ZnOp}$ is $3.22 \mathrm{eV}$. For ZnOsp $E_{g}=3.37 \mathrm{eV}$ (insert in figure 3, a), i.e., do not different from $\mathrm{Eg}$ for a single crystal $\mathrm{ZnO}$. Accordingly, the value of Urbach energy, which is a measure of disorder patterns, is almost twice in ZnOp $\left(E_{o}=0.45 \mathrm{eV}\right)$ than in ZnOsp $\left(E_{o}=0.29 \mathrm{eV}\right)$. As you can see in the AFM images of the surface of $\mathrm{ZnOp}$ and $\mathrm{ZnOsp}($ figure 4) the diameters of the nanorods of the two samples are in the range from 300 to $500 \mathrm{~nm}$. This explains large values of the factor of light scattering on the spectrums of $H f(\lambda)$, mainly due to diffuse reflection from arrays of $\mathrm{ZnO}$, especially for $\mathrm{ZnO}$ ð the range of wavelengths that are commensurable with the diameters of the nanorods (insert in figure $3, \mathrm{~b}$ ).

As follows from comparison of threedimensional images of $\mathrm{ZnOp}$ and $\mathrm{ZnOsp}$ and the
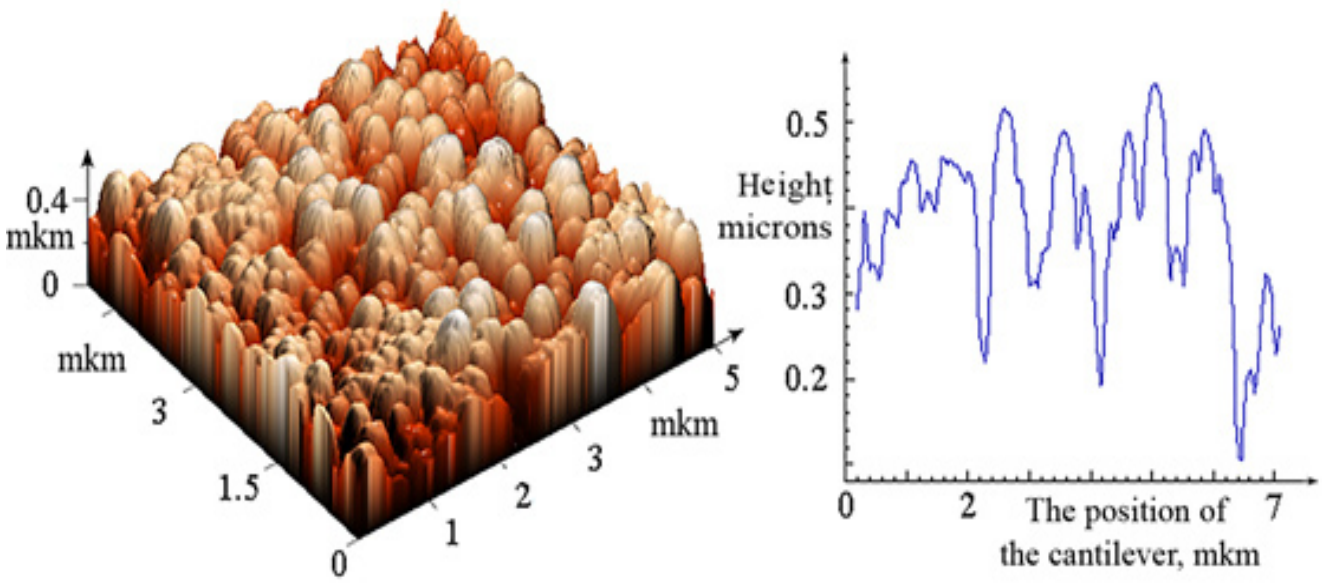

a

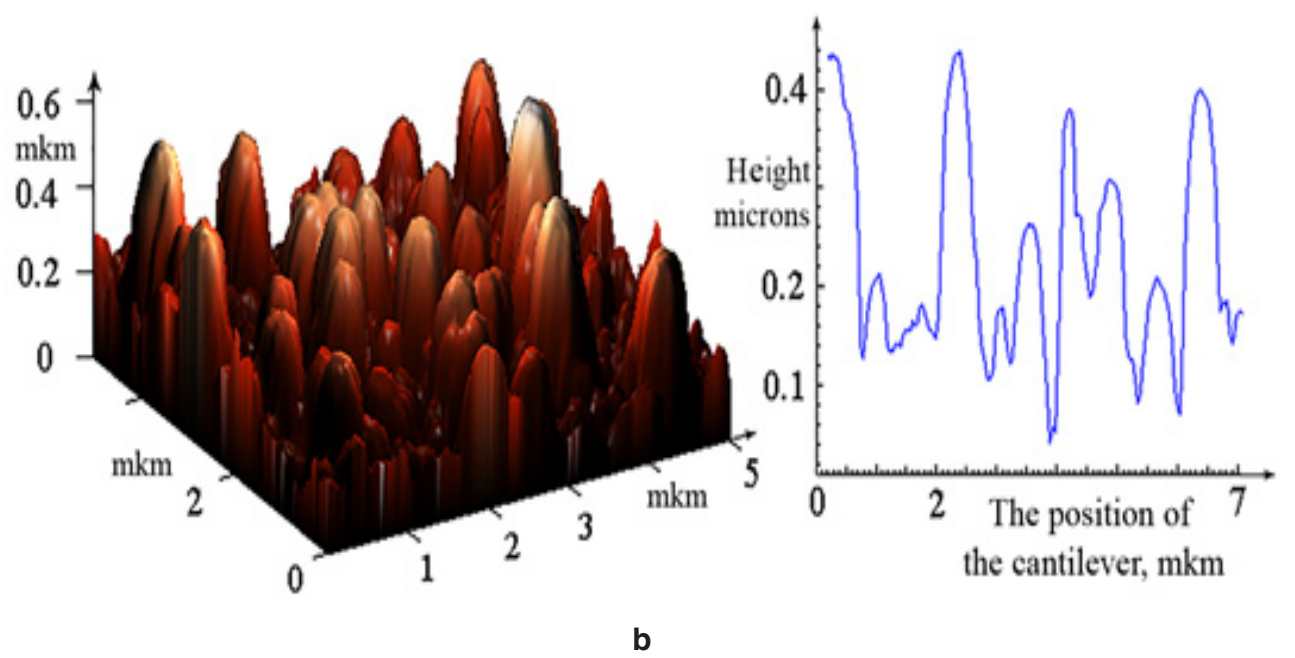

Fig. 4: Three-dimensional AFM images of the surface and the cross-sections (according to AFM) of nanostructured arrays of zinc oxide manufactured without the use of nuclei sublayer (ZnOp) (a) and with the use of nuclei sublayer (ZnOsp) (b). 
corresponding profiles of the cross section of these arrays (figure 4), using the stages of potentiostatic formation of the nanograin sublayer before the pulsed electrodeposition of zinc oxide contributes to the growth of free-standing nanorods of this material, while without such a sublayer of $\mathrm{ZnO}$ the nanorods are packed very tightly and, as a rule, are spliced.

A measurement method of using a thermal probe showed that both samples of zinc oxide which were electrodeposited in pulsed mode have a typical conductivity for ZnO n-type. According to ${ }^{1,18-19}$, the $n$-type of $\mathrm{ZnO}$ conductivity may be due to the presence in its own crystal structure of native defects, in the first place, the non-structure defects of $Z n_{0}$, oxygen vacancies $V_{o}$ and the interstitial $Z n_{i}$. In addition, as indicated in ${ }^{18}$, the $n$-type films of zinc oxide may be caused by their unintentional doping by the interstitial hydrogen $\mathrm{H}_{\mathrm{i}}$, which acts as a shallow donor with an activation energy of about $30 \mathrm{MeV}$. $\ln ^{19}$ is described that the energy level of the defect of $\mathrm{H}_{\mathrm{i}}$ in $\mathrm{ZnO}$ is $0.03-0.05 \mathrm{eV}$.
As it was shown $\mathrm{in}^{10}$, a cathodic reaction of a water recovery with hydrogen release at the potentials that are rather more negative than $-1.05 \mathrm{~V}$ (Rel. Ag/AgCl) leads to the process of electrodeposition of $\mathrm{ZnO}$, allowing more likely the intrusion of hydrogen into the crystal lattice of zinc oxide, especially at the stage of forming the nucleation layer at a constant potential of $-1.3 \mathrm{~V}$ (Rel. $\mathrm{Ag} / \mathrm{AgCl})$. Figure 5 shows the ohmic nature of the dark CVC for both samples.

Calculations of the resistivity of $\mathrm{ZnO}$ gave the higher values of $p_{Z n o}$ for ZnOsp compared to $\mathrm{ZnOp}, 1 \cdot 10^{5}$ and $3 \cdot 10^{4} \Omega \cdot \mathrm{cm}$, respectively, that is possibly associated with a reduced conductivity of nanograion sublayer, that contributed to the average length of nanorod value $p_{Z n O}$. The another probable reason of differences in a specific resistance of the samples $\mathrm{ZnOsp}$ and $\mathrm{ZnOp}$ can be $\mathrm{b}^{1-15,18}$ the anisotropy of electrical properties of polar planes, which include the (002) and nonpolar planes of the hexagonal lattice of the wurtzite. As noted above,

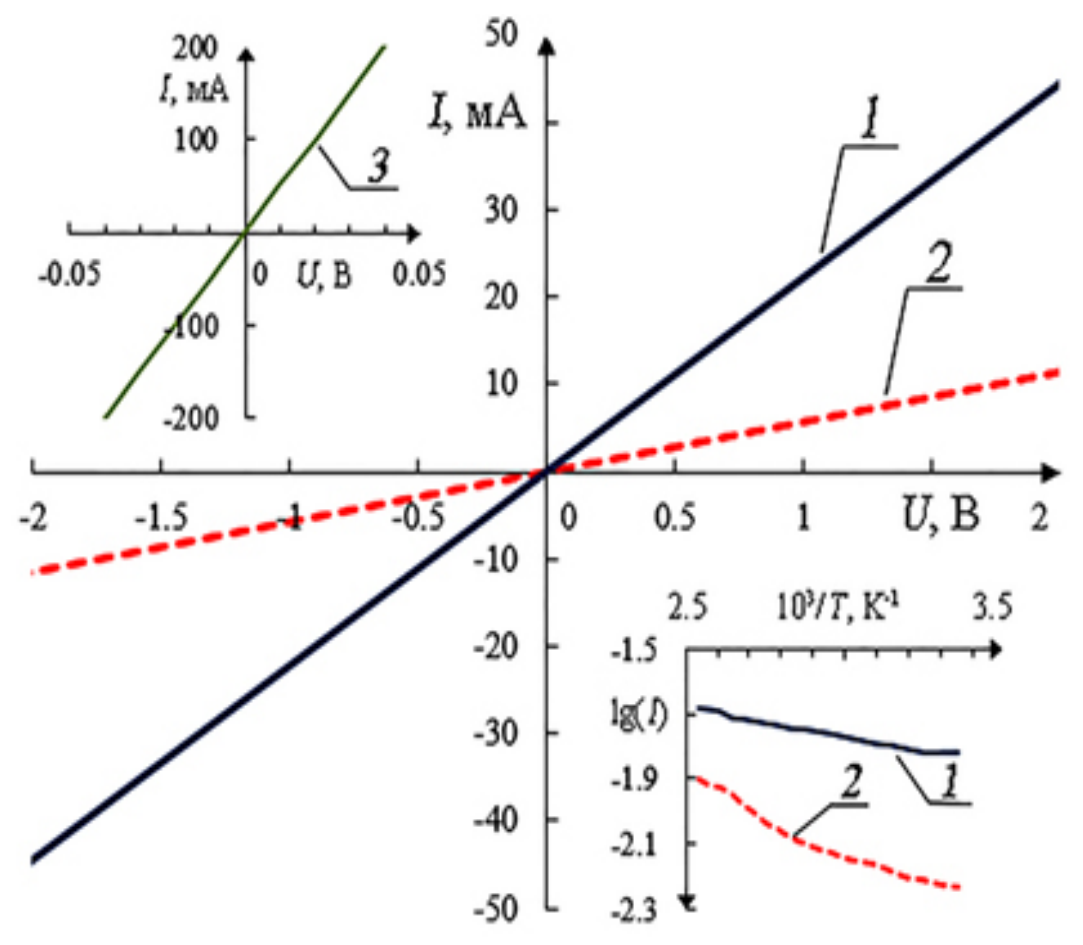

Fig. 5: The dark CVC of nanostructured arrays of zinc oxide, which were electrodeposited without the use of nuclei sublayer (ZnOp) and with the use of the nuclei sublayer (ZnOsp). Upper insert dark CVC of FTO. The bottom inset is the temperature dependence of the electrical conductivity of the respective samples of zinc oxide. 
our structural studies showed that ZnOsp has a much better texture (002) compared with ZnOp, and, consequently, a relatively larger number of polar planes at its surface. The third likely reason of the differences in conductivity of the samples ZnOsp and $\mathrm{ZnOp}$ is the width of the surface depletion region in $\mathrm{ZnO}$, which is caused by adsorption of oxygen from the atmosphere and depends upon the texture of this material, and, in addition, depends on the surface morphology of nanostructured $\mathrm{ZnO}$ arrays ${ }^{4,15}$. The bottom inset in the figure 5 shows the activation energy $E_{\alpha}$, that was defined using a dependency of $\mathrm{lg} /$ from $1 / \mathrm{T}$, and it amounted of $E_{\alpha}=0.04 \mathrm{eV}$ for ZnOp and $E_{\alpha}=0.06 \mathrm{eV}$ for ZnOsp. According to ${ }^{1,15,18-19}$, such activation energy indicates the presence in both samples of fully ionized at a room temperature shallow donor levels of $\mathrm{Zn}_{\mathrm{i}}$ and $\mathrm{H}_{\mathrm{i}}$.

\section{CONCLUSION}

In this work, we demonstrated the influence of $\mathrm{ZnO}$ nanograin sublayers which are created at the initial stage of electrodeposition, on the morphology, structure, optical and electrical properties of nanostructured arrays of 1-D $\mathrm{ZnO}$, produced by the pulsed electrodeposition. It was shown that the formation of such sublayers contributes to the coarsening of crystal grains in the 1-D ZnO and the formation of separate zinc oxide nanorods, arranged perpendicularly to the surface of the FTO substrate. In addition, the electrodeposited array of zinc oxide in a pulsed mode using nanograin sublayers has a higher resistivity, better transparency, less scatters visible light and has a characteristic optical width of the forbidden zone for this material.

\section{ACKNOWLEDGEMENT}

This article was prepared as a part of the implementation of applied research and experimental development (ARED) on the Agreement on the subvention (2015, October, 27) No. 14.579.21.0113 with the financial support of the Ministry of education and science of the Russian Federation. A unique identifier of ARED - RFMEFI57915X0113.

\section{REFERENCES}

1. Litton, C.W.; Reynolds, D.C.; Collins, T.C. Zinc oxide materials for electronic and optoelectronic device applications ., United Kingdom: John Wiley \& Sons, Ltd., 2011.

2. Inamdar, A.I.; Mujawar, S.H.; Sadale, S.B.; Sonavane, A.C.; Shelar, M.B.; Shinde, P.S; Patil, P.S. Electrodeposited zinc oxide thin films: Nucleation and growth mechanism ., Solar Energy Materials \& Solar Cells. 2007, 91, 864-870.

3. Tolosa, M.D.R.; Orozco-Messana, J.; Lima, A.N.C.; Camaratta, R.; Pascual, M.; Hernandez-Fenollosa, M.A. Ålectrochemical deposition mechanism for $\mathrm{ZnO}$ nanorods: diffusion coefficient and growth models., Journal of The Electrochemical Society. 2011, 158, E107-E110.

4. Skompska, M.; Zarêbska, K. Electrodeposition of $\mathrm{ZnO}$ nanorod arrays on transparent conducting substrates-a review., Electrochimica Acta. 2014, 127, 467-488.
5. Klochko, N.P.; Khrypunov, G.S.; Myagchenko, Y.O.; Melnychuk, E.E.; Kopach, V.R.; Klepikova, E.S.; Lyubov, V.M.; Kopach, A.V. Controlled growth of one-dimensional zinc oxide nanostructures in the pulsed electrodeposition mode., Semiconductors. 2012, 46, 825-831.

6. Klochko, N.P.; Klepikova, K.S.; Tyukhov, I.I.; Myagchenko, Y.O.; Melnychuk, E.E.; Kopach, V.R.; Khrypunov, G.S.; Lyubov, V.M.; Kopach, A.V. Structure and optical properties of sequentially electrodeposited $\mathrm{ZnO} / \mathrm{Se}$ bases for ETA solar cells ., Solar Energy. 2015, 120, 330-336.

7. Klochko, N. P.; Klepikova, E. S.; Khripunov, G. S.; Volkova, N.D.; Kopach, V. R.; Lyubov, V. N.; Kirichenko, M. V.; Kopach, A. V. Antireflection nanostructured arrays of zinc oxide fabricated by pulsed electrodeposition ., Physics and technics of semiconductors. 2015, 49, 219229. 
8. Riedel, W.; Tang, Y.; Ohm, W.; Chen, J.; LuxSteiner, M.Ch.; Gledhill, S. Effect of initial galvanic nucleation on morphological and optical properties of $\mathrm{ZnO}$ nanorod arrays ., Thin Solid Films 2015, 574, 177-183.

9. Klochko, N.P.; Klepikova, K.S.; Tyukhov, I.I.; Myagchenko, Y.O.; Melnychuk, E.E.; Kopach, V.R.; Khrypunov, G.S.; Lyubov, V.M.; Kopach, A.V.; Starikov, V.V.; Kirichenko, M.V. Zinc oxide-nickel cermet selective coatings obtained by sequential electrodeposition ., Solar Energy. 2015, 117, 1-9.

10. Klochko, N.P.; Khrypunov, G.S.; Myagchenko, Y.O.; Melnychuk, E.E.; Kopach, V.R.; Klepikova, K.S.; Lyubov, V.M.; Kopach, A.V. Electrodeposited zinc oxide arrays with the moth-eye effect., Semiconductors. 2014, 48, 531-537.

11. Tsybulya, S. V. Introduction to the structural analysis of nanocrystals. Novosibirsk: Novosibirsk state University, 1980.

12. Kuz'micheva, G. M. X-ray of nano-sized objects. Part I MITHT. Training manual. Moscow: MITHT them. M. Lomonosov, 2010.
13. Palatnik, L. S. Structure and physical properties of solid objects. Laboratory workshop, Kiev: Vishcha school, 1983.

14. Axelevitch, A.; Golan, G. Hot-probe method for evaluation of majority charged carriers concentration in semiconductor thin films. Facta Universitatis Series: Electronics and Energetics, 2013.

15. Brillson, L.J.; Lu, Y. ZnO Schottky barriers and ohmic contacts .,J. Appl. Phys. 2011, 109, 121301-1-121301-33.

16. Mazda, F.F. Electronic Instruments and Measurement Techniques. Cambridge: Cambridge University Press, 1987.

17. Srivastava, R. Investigation on temperature sensing of nanostructured zinc oxide synthesized via oxalate route ., Journal of Sensor Technology, 2012, 2, 8-12.

18. Morkoç, H.; Özgür, Ü. Zinc Oxide: Fundamentals, Materials and Device Technology. Weinheim: WILEY-VCH Verlag GmbH \& Co. KGaA, 2009.

19. Djuri, A.B.; Ng, A.M.C.; Chen, X.Y. ZnO nanostructures for optoelectronics: Material properties and device applications ., Progress in Quantum Electronics. 2010, 34, 191-259. 